\title{
Association between latent toxoplasmosis and clinical course of schizophrenia - continuous course of the disease is characteristic for Toxoplasma gondii-infected patients
}

\author{
${ }^{1}$ Department of Parasitology, Faculty of Medicine, Adiyaman University, Adiyaman, Turkey; \\ ${ }^{2}$ Department of Psychiatry, Faculty of Medicine, Inonu University, Malatya, Turkey; \\ ${ }^{3}$ Malatya State Hospital, Department of Psychiatry, Malatya, Turkey; \\ ${ }^{4}$ Department of Parasitology, Faculty of Medicine, Ankara University, Ankara, Turkey; \\ ${ }^{5}$ Department of Biostatistics, Inonu University, Malatya, Turkey
}

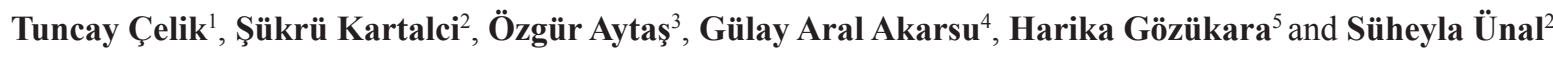

\begin{abstract}
The aim of the present study was to investigate the association between various clinical aspects of schizophrenia and seropositivity against Toxoplasma gondii (Nicolle et Manceaux, 1908). We selected 94 patients with schizophrenia and investigated the seropositivity rate for anti-T. gondii IgG antibodies by ELISA. Clinical parameters of schizophrenic patients such as illness type and status, clinical course, awareness of the illness and need for electroconvulsive therapy (ECT) were compared with their serological status. Anti-T. gondii IgG antibodies were detected in 43 (46\%) of schizophrenic patients. Chronic patients had a rate of 34 (72\%) seropositivity, whereas $9(22 \%)$ of the patients with partial remission showed evidence of latent toxoplasmosis. Of continuous patients, 35 $(81 \%)$ were found to be seropositive and this rate was significantly more than in the other groups. The rate of latent toxoplasmosis was detected significantly higher in patients who lack awareness of schizophrenia (36, i.e. $72 \%$ ) than the patients who were aware of their illnesses (7, i.e. 16\%). Anti-T. gondii IgG antibodies were detected in 38 (70\%) of ECT performed patients while this percentage was $13 \%$ in the ones who had never been treated with ECT. This difference was also statistically significant. We showed that Toxoplasmainfected subjects had $15 \times$ higher probability of having continuous course of disease than Toxoplasma-free subjects. Our results put forth the possibility of latent toxoplasmosis to have a negative impact on the course of schizophrenia and treatment response of schizophrenic patients.
\end{abstract}

Keywords: toxoplasmosis, schizophrenia patients, prognosis, serology

Toxoplasma gondii (Nicolle et Manceaux, 1908) is an intracellular apicomplexan parasite that can infect a wide range of hosts including humans. It can be contracted by humans through exposure to soil, water, undercooked meat and cat faeces that contain various life cycle stages of the parasite (Jones et al. 2001). It can invade and multiply in almost every cell type in its host. It stays dormant in tissue cysts consisting of thousands of bradyzoites and these tissue cysts play a key role in the life cycle of the protist. The tissue cysts have been shown to cause significant and continuous damage to neural tissues in rodents (Vyas et al. 2007).

Toxoplasma gondii can also reach human central nervous system as a result of its permissibility (Carruthers and Suzuki 2007, Torrey et al. 2007), and remain latent as tissue cysts. There is increasing evidence demonstrating that the localisation of these latent forms within the central nervous system is associated with mood and behaviour al- teration in humans (Yolken et al. 2009). As such, studies are currently focusing on the possible relationship between chronic toxoplasmosis and behavioural pathologies as well as chronic illnesses of the central nervous system (Yolken et al. 2009).

The presence of latent tissue cysts in the brain is believed to cause changes in its functioning that potentially leads to schizophrenia and other neurological disorders in humans. This is engendered by the compression damage caused onto brain tissue, as well as the alteration of brain physiology due to the effects on neurotransmitter activity (Henriquez et al. 2009, Webster and McConkey 2010, Goodwin et al. 2011, Strobl et al. 2012). A standard computer-based assessment, which was employed to compare reaction times between infected and uninfected individuals, demonstrated that infected individuals had lower performance and concentration levels than uninfected individuals (Novotná et al. 2008). 
Schizophrenia is a neuropsychiatric disorder with a worldwide prevalence of 4-7/1000 persons and with a devastating impact on patients and their families. There are various factors influencing the incidence and prevalence of schizophrenia such as sex, urbanicity and migrant status. Although the prevalence of the illness is lower in developing countries, there is no evidence to suggest that the incidence differs due to economic status of the nations (McGrath et al. 2004, Saha et al. 2005, 2006, Yolken et al. 2009). Development of schizophrenia is mainly associated with genetic and environmental factors like exposure to pathogens, physical stress and faetal starvation during pregnancy, being known as a factor that increases individuals' predisposition (Susser and Lin 1992, Yolken et al. 2001). An immune basis for the illness, including mutations in the immune system that contributes to the alteration of the neurotransmitter physiology, has also been proposed Muller and Schwarz (2006). Other studies have shown that patients with schizophrenia experience structural and physiological changes in their brains even when they have never been treated with antipsychotics (Torrey 2002).

Psychotic symptoms involving delusions and hallucinations can be observed in patients with acute toxoplasmosis and AIDS patients with reactivated $T$. gondii infections. Such symptoms, however, are sometimes misinterpreted as symptoms of schizophrenia (Wang et al. 2006). Although T. gondii seropositivity is more frequent among schizophrenic patients, it is still not apparent either it is a cause or a consequence. Some studies strongly suggest that infection with $T$. gondii is a significant risk factor for the development of schizophrenia (Torrey and Yolken 2003, Cetinkaya et al. 2007, Henriquez et al. 2009, Torrey et al. 2012, Flegr 2013, Flegr et al. 2014).

To date, only a few studies have been undertaken about the effects of latent toxoplasmosis on the clinical characteristics of the schizophrenic patients. In a preliminary prevalence study, a relationship between seropositivity and a greater cognitive impairment in schizophrenia was found, whereas no significant association between serological status and Positive and Negative Syndrome Scale (PANSS) scores (Kay et al. 1987; Boronov et al. 2002). Wang et al. (2006) reported that seropositive schizophrenic patients had higher scores of the positive, cognitive and excitement components and lower scores of negative component of PANSS than the seronegative ones. A more recent finding indicates that latent toxoplasmosis reduces gray matter density in schizophrenia but not in controls (Horáček et al. 2012). Lately, it was reported that T. gondiiinfected patients spent more days than the uninfected ones in the hospital during their last stay. Also, their mean daily doses of antipsychotics were higher. They concluded that latent toxoplasmosis in schizophrenia may lead to more severe positive psychopathology and perhaps less favourable course of schizophrenia (Holub et al. 2013).

The association between schizophrenia and toxoplasmosis has been investigated in many studies, but the impact of this association on the prognosis of the illness is not enlightened. The aim of the present study was to investigate the association between latent infection with $T$. gondii and the clinical course in schizophrenic patients. We hypothesised that toxoplasmosis has a negative impact on the prognosis and treatment of schizophrenic patients.

\section{MATERIALS AND METHODS}

\section{Patients and clinical measure}

Patients consisted of 94 individuals who were admitted to the Department of Psychiatry at Inonu University Faculty of Medicine, and a nursing home in Malatya, Turkey with the diagnosis of schizophrenia in 2011 and 2012. Among patients, 32 (34\%) were women and $62(66 \%)$ were men and their median age was 40 years (range, 17-90 years; standard deviation 16 years). At the time of admission, none of the patients showed clinical evidence of immunodeficiency.

Clinical schizophrenia diagnoses were established by two psychiatrists upon the Turkish version of the Structured Clinical Interview for the Structured Clinical Interview for DSM-IV (Çorapcioğlu et al. 1999). The subjects from the university hospital were follow-up patients who were not in a psychotic episode, whereas the residents of the nursing home were chronic patients requiring medical care. Some clinical data on the patients such as illness status, clinical course, awareness of the illness, electroconvulsive therapy, compliance with medication and illness type were chosen as indicators of prognosis to compare with the antiToxoplasma gondii serological status. All the patients were recorded in respect to illness status (chronic, partially cured, cured to a great extent), clinical course (first episode, single past episode and full remission, single past episode and partial remission, episodic without residual symptoms between episodes, episodic with residual symptoms between episodes, continuous), awareness of the illness (absent, present), electroconvulsive therapy (ECT) (performed, not performed), compliance with medication (not compliant, irregular or applies by persuasion, irregular with help, regular by self), illness type (catatonic, disorganised, paranoid, residual and undifferentiated). They all agreed to participate in the study. Written informed consents were obtained from all of the subjects after the study procedure had been explained. The study protocols and the consent forms were approved by Inonu University Ethics Committee.

\section{Serum collection and laboratory assay}

Latent toxoplasmosis is a life-long illness in humans and, therefore, the presence of anti- $T$. gondii $\operatorname{IgG}$ antibodies is considered as a reliable indicator of viable bradyzoites in tissue cysts that persist in infected individuals (Montoya and Liesenfeld 2004). For this reason, the blood samples were obtained from the patients by venipuncture to investigate anti- $T$. gondii IgG antibodies. Sera were separated from whole blood and were stored at $-80^{\circ} \mathrm{C}$ until tested. The levels of class specific immunoglobulin $\mathrm{G}(\mathrm{IgG})$ antibodies against purified $T$. gondii antigens in serum samples were measured using enzyme-linked immunosorbent assay (ELISA) performed according to manufacturer instructions (Cobas Core, Roche, Langen, Germany).

\section{Statistical analysis}

The data are represented as count and percentage. To compare the groups according to T. gondii seropositivity, Pearson chi-square and likelihood ratio tests were used. When the as- 
Table 1. The relationship between toxoplasmosis and continuous course of schizophrenia determined by using a logistic regression with dependent variable continuous course and independent variable toxoplasmosis.

\begin{tabular}{lcccc}
\hline Factor & Toxoplasmosis & Age & Sex & Population \\
\hline $\mathrm{t}(89)$ & 4.318 & 1.387 & -0.532 & 2.813 \\
$\mathrm{P}$ & $<0.001$ & 0.169 & 0.596 & 0.006 \\
O. R. (C. I. 95) & $15.0(4.3-52.2)$ & $11.6(0.35-392.5)$ & $0.71(0.20-2.56)$ & $6.46(1.73-24.13)$ \\
\hline
\end{tabular}

$\mathrm{t}$ - t-value; P - P-value; O. R. - odds ratio; C. I. - Confidence Interval.

Table 2. Comparison of various clinical parameters of schizophrenia with serological status against Toxoplasma gondii.

\begin{tabular}{|c|c|c|c|c|c|}
\hline \multirow{2}{*}{\multicolumn{2}{|c|}{ Clinical parameters }} & \multirow{3}{*}{$\begin{array}{c}\text { Total count } \\
47\end{array}$} & \multicolumn{2}{|c|}{ Anti-Toxoplasma $\operatorname{IgG}$} & \multirow{3}{*}{$\mathrm{P}$} \\
\hline & & & \multirow{2}{*}{$\frac{\text { negative C }(\%)}{13(28)}$} & \multirow{2}{*}{$\frac{\text { positive } \mathrm{C}(\%)}{34(72)}$} & \\
\hline & chronic* & & & & \\
\hline Illness status & partial remission & 41 & $32(78)$ & $9(22)$ & $<0.001$ \\
\hline & remission to a great extent & 6 & $6(100)$ & $0(0)$ & \\
\hline \multirow{6}{*}{ Clinical course } & first episode & 1 & $1(100)$ & $0(0)$ & \multirow{6}{*}{$<0.001$} \\
\hline & single past episode and full remission & 2 & $2(100)$ & $0(0)$ & \\
\hline & single past episode and partial remission & 10 & $9(90)$ & $1(10)$ & \\
\hline & episodic without residual symptoms inbetween & 15 & $12(80)$ & $3(20)$ & \\
\hline & episodic with residual symptoms inbetween & 23 & $19(83)$ & $4(17)$ & \\
\hline & continuous* & 43 & $8(19)$ & $35(81)$ & \\
\hline \multirow{2}{*}{ Illness awareness } & present & 44 & $37(84)$ & $7(16)$ & \multirow{2}{*}{$<0.001$} \\
\hline & absent & 50 & $14(28)$ & $36(72)$ & \\
\hline \multirow{2}{*}{ ECT } & performed & 54 & $16(30)$ & $38(70)$ & \multirow{2}{*}{$<0.001$} \\
\hline & not performed & 40 & $35(88)$ & $5(13)$ & \\
\hline \multirow{2}{*}{$\begin{array}{l}\text { Compliance with } \\
\text { medication }\end{array}$} & with help & 83 & $42(51)$ & $41(49)$ & \multirow{2}{*}{0.051} \\
\hline & by self & 11 & $9(82)$ & $2(18)$ & \\
\hline \multirow{5}{*}{ Illness type } & paranoid & 41 & $24(59)$ & $17(42)$ & \multirow{5}{*}{0.611} \\
\hline & undifferentiated & 10 & $4(40)$ & $6(60)$ & \\
\hline & residual & 12 & $6(50)$ & $6(50)$ & \\
\hline & disorganized & 17 & $11(65)$ & $6(35)$ & \\
\hline & catatonic & 14 & $6(43)$ & $8(57)$ & \\
\hline
\end{tabular}

* shows the statistically significant group due to the post-hoc analysis; ECT - Electroconvulsive Therapy.

sumptions of the asymptotic method could not be met, the exact significance of Pearson chi-square test was used. We used binary logistic regression analysis for estimation of odds ratios and their confidence intervals. In all analysis we considered the significance level as 0.05 .

\section{RESULTS}

In the present study, 43 of the 94 (46\%) cases with schizophrenia were positive for anti-Toxoplasma gondii IgG antibodies. There was no significant difference between genders in respect to the seropositivity rate $(\mathrm{P}=0.874)$. Anti-T. gondii IgG was detected in the sera of $33(60 \%)$ out of 55 patients from the nursing home, whereas $10(26 \%)$ of 39 patients from the university hospital were seropositive. This difference was significant $(\mathrm{P}=0.001 ; \mathrm{OR}=4.4 ; 95 \%$ C.I. $=1.8-10.7)$.

Results of comparison of various clinical parameters of schizophrenia with serological status against $T$. gondii are presented in Table 1. Considering the seropositivity in groups with different illness status, T. gondii seropositivity rate among 'chronic' patients was found to be $72 \%$ $(\mathrm{OR}=9.3 ; 95 \%$ C.I. $=3.5-24.7)$ whereas it was $22 \%$ in partial remission group and no case was positive in remission to a great extent group. Prevalence of toxoplasmosis detected in patients with chronic illness was significantly higher than the ones in partial remission and in remission to a great extent groups $(\mathrm{P}<0.001)$.

When the relationship between the clinical course of the illness and past $T$. gondii infection was examined, we observed that $T$. gondii infection was significantly more prevalent among the 'continuous' group than the others $(\mathrm{P}<0.001)$. No T. gondii seropositivity was detected among the 'first episode' or 'single past episode and full remission' groups. When the 'single past episode and partial remission' group with 1 (10\%) seropositivity was referred, $3(20 \%)(\mathrm{P}=0.512$; OR $=2.3$; 95\% C.I. $=0.2-25.4)$ patients of the 'episodic without residual symptoms between episodes' group and $4(17 \%)$ patients of the 'episodic with residual symptoms between episodes' group had $T$. gondii infection. In contrast, 35 (81\%) $(\mathrm{P}<0.001$; OR = 39.4; $95 \%$ C.I. $=44.0-356.8)$ of the 'continuous' patients had anti-T. gondii $\mathrm{IgG}$ antibodies.

Since much higher frequency of $T$. gondii-infected subjects $(81 \%)$ was observed in subset of 43 patients with continuous course of disease than in other 51 subjects $(16 \%)$, we coded this form of disease with a binary variable 'continuous' and analysed differences between patients with and without continuous course of disease using a logistic 
regression with dependent variable continuous and independent variables toxoplasmosis, age, sex and population. The results showed that $T$. gondii-infected subjects had fifteen times higher probability of having continuous course of disease than T. gondii-free subjects (Table 1).

We also investigated the relationship between the patients' awareness of schizophrenia and toxoplasmosis seroprevalence in the study group. While anti-T. gondii IgG antibodies were detected in $7(16 \%)$ of the patients with awareness, this rate increased to $36(72 \%)$ among patients without awareness of the illness. This difference was significant $(\mathrm{P}<0.001 ; \mathrm{OR}=13.6 ; 95 \%$ C.I. $=4.9-37.6)$.

Additionally, the association between ECT and past T. gondii infection was also found to be significant $(\mathrm{P}<0.001 ; \mathrm{OR}=16.6 ; 95 \%$ C.I. $=5.5-50.2)$. The rate of seropositivity among ECT performed patients was 38 (70\%), whereas it was $5(13 \%)$ for patients to whom ECT was never performed. There was no significant difference between seropositive and seronegative groups in respect to treatment compliance $(\mathrm{P}=0.051)$.

The types of schizophrenia in the study group were catatonic $(n=8)$, disorganised $(n=6)$, paranoid $(n=17)$, residual $(n=6)$ and undifferentiated $(n=6)$. The toxoplasmosis frequency among patients was compared with types of schizophrenia but no significant difference was observed $(\mathrm{p}=0.611)$. Apart from these findings, three patients with latent toxoplasmosis also had intellectual disabilities. The relationship between $T$. gondii infection and the abovementioned criteria is shown in Table 2 .

Theoretically, patients in the nursing home could have both increased probability of $T$. gondii infection and more serious course of schizophrenia. This could result in observed associations between toxoplasmosis and various variables describing seriousness of schizophrenia disease. To test this hypothesis, we performed logistic and ordinal regressions with independent variables toxoplasmosis, age, sex and population (nursing home yes/no) for binary (ECT, awareness) and ordinal (course, compliance, illness status) variables, respectively. The effects of toxoplasmosis remained significant, except for the compliance with medication (ECT: $\mathrm{P}<0.001$; illness awareness: $\mathrm{P}<0.001$; clinical course: $\mathrm{P}<0.001$; compliance with medication: $\mathrm{P}=0.993$; illness status: $\mathrm{P}=0.008)$.

\section{DISCUSSION}

Schizophrenia, like multiple sclerosis and Parkinson's disease, is a chronic illness of the central nervous system and as such, infectious agents can also be blamed as a potential etiological factor, perhaps in persons who also have an increased genetic susceptibility (Torrey and Yolken 2003, Çelik et al. 2013). In recent years, serological studies on patients with schizophrenia have been carried out showing that the rate of anti-Toxoplasma gondii antibodies were higher in patients than in all the selected control groups (Yolken et al. 2001, Çetinkaya et al. 2007, Tamer et al. 2008). We did not compare seropositivity rate in the study group with a healthy control group, which was out of the range of this study, but the overall anti-Toxoplasma IgG positivity in our patient study group was $46 \%$ and the difference between the seropositivity rates of two gender was not statistically significant. This value is slightly higher the seroprevalance rate reported from this region of Turkey, which is around $40 \%$ (Çelik et al. 2010, Doğan et al. 2012).

There have been only a few studies investigating the association between seropositivity against $T$. gondii and symptoms in schizophrenic patients. In one of these studies, a significant relationship between severe positive schizophrenia symptoms in ultra-high-risk individuals for psychosis and higher levels of anti-T. gondii IgG antibodies was observed (Amminger et al. 2007). Following this study, more severe positive, disorganised and excitement symptoms among schizophrenic patients with latent toxoplasmosis were reported (Holub et al. 2013). In the present study, while most of the chronic schizophrenic patients (72\%) were seropositive, none of the treatment responsive patients had specific antibody against $T$. gondii. We interpret this finding as latent toxoplasmosis could have a negative impact on clinical course of schizophrenia. According to a previous studies, behavioral changes in $T$. gondii-infected individuals are likely to be related to increases in the levels of dopamine, a neurotransmitter that is assumed to play a significant role in schizophrenia (Skallová et al. 2006, Gaskell et al. 2009, Prandovszky et al. 2011, Hodková et al. 2007). Such elevations in dopamine concentrations possibly contribute to the worsening and deterioration of schizophrenia symptoms.

In the present study, anti- $T$. gondii $\operatorname{IgG}$ positivity among patients from the nursing home was $4.3 \times$ higher than the patients from the university hospital. The patients at the nursing home were unable to care for themselves and the onset time of schizophrenia could not be detected. As they were older than the ones from the university hospital, all the data were adjusted with age. It may be assumed that the patients at the nursing home possess bad hygiene practices and nutrition status and thus was easier for them to acquire toxoplasmosis. However, latent toxoplasmosis may also be considered to have a negative impact on the treatment of schizophrenia and quality of life. A continuous clinical course was observed fifteen times higher among seropositive patients, which was a finding independent from age, sex and population. Therefore, we propose that latent toxoplasmosis may affect the response to the schizophrenia treatment, prolong hospital stay and as a result increase the cost of the illness.

Among schizophrenia patients, the insight is a multifaceted concept that includes the following aspects: awareness of the mental illness, awareness of symptoms, awareness of social consequences and awareness of the effects of treatment. Awareness levels are reported to be related to the severity and extent of the patient's symptoms (Lysaker et al. 1994). We investigated the awareness of illness as a subset of insight and our findings showed that latent toxoplasmosis was more prevalent $(72 \%)$ among patients who lacked awareness. Therefore, we thought that absence of awareness showing a severe illness was related to specific $T$. gondii-IgG positivity. 
ECT is still considered to be one of the effective therapeutic approaches, especially for the patients who has insufficient or partial response to medication (Abrams 2002). Anti-T. gondii IgG antibodies were detected in $70 \%$ of patients to whom ECT was performed. We think that the possible negative effect of latent toxoplasmosis on the clinical outcome of schizophrenia may have increased the need for ECT in these patients.

Based on our results, we identified a negative correlation between the clinical picture of schizophrenia and the presence of anti-T. gondii $\mathrm{IgG}$ antibodies in patients with latent toxoplasmosis. This is indicative of the fact that the presence of $T$. gondii may lead to some changes in the psychopathology, and also that $T$. gondii infections may be associated with worse prognosis among patients with schizophrenia. This observation can be considered as a reflection of the effect of $T$. gondii infections on schizophrenia's psychopathology in different individuals, and also of the cumulative effect that these infections have over time on the patients' symptoms.

The strongest aspect of the present study is that we compared the relationship between latent toxoplasmosis and the subtypes and clinical course of this illness with het- erogenous forms, although the previous studies compared the relationship between toxoplasmosis and the severity of the illness. The major limitation of this study is the small size of the study group, which did not allow for interpretation of the whole dataset. Although schizophrenia is familial, environmental factors such as toxoplasmosis should be investigated in order to understand why the illness has various clinical courses. Therefore, new studies with greater sample sizes regarding this issue should be carried out.

As a result, there seems to be a relation between latent toxoplasmosis and the course of schizophrenia and treatment response. We assume that the effects of toxoplasmosis on schizophrenic patients should be further investigated for a longer period of time. Following future studies, novel treatment regimens might be considered to improve the prognosis of schizophrenia in seropositive patients.

Acknowledgements. We are grateful for the kind comments of the reviewers. We are thankful for the help of physicians at the Department of Psychiatry, School of Medicine, Inonu University and at the Department of Psychiatry, Malatya State Hospital. The work was financed by authors who declare that they have no conflict of interest.

\section{REFERENCES}

AbRAms R. 2002: Electroconvulsive therapy (ECT) practice in Metropolitan New York community hospitals. Psychol. Med. 32: 1323-1324.

Amminger G.P., McGorry P.D., Berger G.E., Wade D., Yung A.R., Phillips L.J., Harrigan S.M., Francey S.M., Yolken R.H. 2007: Antibodies to infectious agents in individuals at ultra-high risk of psychosis. Biol. Psych. 61: 1215-1217.

Boronow J., Dickerson F., Stallings C., Lee B., Origoni A., Yolken R. 2002: HSV-1, CMV and Toxoplasma serology predict cognitive defcits in schizophrenia. Schizophr. Res. 53: 85-89.

Carruthers V.B., Suzuki Y. 2007: Effects of Toxoplasma gondii infection on the brain. Schizophr. Bull. 33: 745-751.

Çelik T., Kamişli O., Babür C., Çevik M.Ö., Öztuna D., AlTINAYAR S. 2010: Is there a relationship between Toxoplasma gondii infection and idiopathic Parkinson's disease? Scand. J. Infect. Dis. 42: 604-608.

Çelik T., Kaplan Y., Ataş E., Öztuna D., Berilgen S. 2013: Toxocara seroprevalence in patients with idiopathic Parkinson's disease: chance, association or coincidence? Biomed. Res. Int.: 685196.

Çetinkaya Z., Yazar S., Gecici O., Namli M.N. 2007: AntiToxoplasma gondii antibodies in patients with schizophreniapreliminary findings in a Turkish sample. Schizophr. Bull. 33: 789-791.

Çorapcioğlu A., Aydemir O., Yildiz M., Esen A., Koroglu E. 1999: [Structured clinical interview for DSM-IV axis I disorders (SCID-I), clinical version, Ankara.] Physicians broadcasting union. Journal of Drug and Therapeutics. 12: 233-236. (In Turkish.)

DoĞan K., Kafkasli A., Karaman U., Atambay M., KARAOĞLU L., ÇOLAK C. 2012: The rates of seropositivity and seroconversion of Toxoplasma infection in pregnant women, Ankara. Bull. Microbiol. 46: 290-294.

FLEGR J. 2013: How and why Toxoplasma makes us crazy. Trends Parasitol. 29: 156-163.

Flegr J., Príplatová L., Hampl R., Bičíková M., Ripová D., Mohr P. 2014: Difference of neuro- and immunomodulatory steroids and selected hormone and lipid concentrations between
Toxoplasma-free and Toxoplasma-infected schizophrenia patients but not CMV-free and CMV-infected schizophrenia patients. Neuroendocrinol. Lett. 35: 20-27.

Gaskell E.A., Smith J.E., Pinney J.W., Westhead D.R., McCONKey G.A. 2009: A unique dual activity amino acid hydroxylase in Toxoplasma gondii. PLoS ONE 4: e4801.

Goodwin D.G., Strobl J.S., Lindsay D.S. 2011: Evaluation of five antischizophrenic agents against Toxoplasma gondii in human cell cultures. J. Parasitol. 97: 148-151.

Henriquez S.A., Brett R., Alexander J., Pratt J., Roberts C.W. 2009: Neuropsychiatric disease and Toxoplasma gondii infection. Neuroimmunomodulation 16: 122-133.

Hodková H., Kodym P., Flegr J. 2007: Poorer results of mice with latent toxoplasmosis in learning tests: impaired learning processes or the novelty discrimination mechanism? Parasitology 134: 1329-1337.

Holub D., Flegr J., Dragomirecká E., Rodriguez M., Preiss M., Novák T., Čermák J., Horáček J., Kodym P., Libiger J., Höschl C., Motlová L.B. 2013: Differences in onset of disease and severity of psychopathology between toxoplasmosis-related and toxoplasmosis-unrelated schizophrenia. Acta Psychiatr. Scand. 127: 227-238.

Horáček J., Flegr J., Tintěra J., Verebová K., Španiel F., Novák T., BrunovskÝ M., Bubeníková-Valešová V., HoluB D., PÁleníčeK T., Höschl C. 2012: Latent toxoplasmosis reduces gray matter density in schizophrenia but not in controls: voxel-based-morphometry (VBM) study. World J. Biol. Psychiatry 13: 501-509.

Jones J.L., Kruszon-Moran D., Wilson M., McQuillan G., Navin T., McAuley J.B. 2001: Toxoplasma gondii infection in the United States: seroprevalence and risk factors. Am. J. Epidemiol. 154: 357-365.

Kay S.R., Fisbzbein A., Opler L.A. 1987: The Positive and Negative Syndrome Scale (PANSS) for Schizophrenia. Schizophrenia Bulletin. 13: 261-276.

Lysaker P., Bell M., Milstein R., Bryson G., Beam-Goulet J. 1994: Insight and psychosocial treatment compliance in schizophrenia. Psychiatry 57: 307-315. 
McGrath J., Saha S., Welham J., El SaAdi O., Chant D.A. 2004: Systematic review of the incidence of schizophrenia: the distribution of rates and the influence of sex, urbanicity, migrant status and methodology. BMC Med. 2: 1-22.

Montaya J.G., Liesenfeld O. 2004: Toxoplasmosis. Lancet 363 1965-1976.

Muller N., Schwarz M. 2006: Schizophrenia as an inflammation-mediated dysbalance of glutamatergic neurotransmission. Neurotox. Res. 10: 131-148.

Novotná M., Havlíček J., Smith A.P., Kolbeková P., Skallová A., Klose J., Gasová Z., Písacká M., Sechovská M., FLEGR J. 2008: Toxoplasma and reaction time: role of toxoplasmosis in the origin, preservation and geographical distribution of Rh blood group polymorphism. Parasitology. 135: 1253-1261.

Prandovszky E., Gaskell E., Martin H., Dubey J.P., WebSter J.P., MCConkey G.A. 2011: The neurotropic parasite Toxoplasma gondii increases dopamine metabolism. PLoS ONE 6: e23866.

Saha S., Chant D., Welham J., McGrath J. 2005: The systematic review of the prevalence of schizophrenia. PLoS Med. 2: $413-433$

Saha S., Welham J., Chant D., McGrath J. 2006: Incidence of schizophrenia does not vary with economic status of the country. Evidence from a systematic review. Soc. Psychiatry Psychiatr. Epidemiol. 41: 338-340.

Skallová A., Kodym P., Frynta D., Flegr J. 2006: The role of dopamine in Toxoplasma-induced behavioural alterations in mice: an ethological and ethopharmacological study. Parasitology 133: 525-535.

Strobl J.S., Goodwin D.G., Rzigalinski B.A., Lindsay D.S. 2012: Dopamine stimulates propagation of Toxoplasma gondii tachyzoites in human fibroblast and primary neonatal rat astrocyte cell cultures. J. Parasitol. 98: 1296-1299.
SusSER E.S, Lin S.P. 1992: Schizophrenia after prenatal exposure to the Dutch Hunger Winter of 1944-1945. Arch. Gen. Psychiatry. 49: 983-988.

Tamer G.S., Dundar D., Yalug I., Caliskan S., Yazar S., Aker A. 2008: The schizophrenia and Toxoplasma gondii connection: infectious, immune or both? Adv. Ther. 25: 703-709.

TORREY E.F. 2002: Studies of individuals with schizophrenia never treated with antipsychotic medications: a review. Schizophr. Res. 58: 101-115.

Torrey E.F., Bartko J.J., Lun Z.R, Yolken R.H. 2007: Antibodies to Toxoplasma gondii in patients with schizophrenia: a meta-analysis. Schizophr. Bull. 33: 729-736.

Torrey E.F., BARTKo J.J., Yolken R.H. 2012: Toxoplasma gondii and other risk factors for schizophrenia: an update. Schizophr. Bull. 38: 642-647.

Torrey E.F., Yolken R.H. 2003: Toxoplasma gondii and schizophrenia. Emerg. Infect. Dis. 9: 1375-1380.

Vyas A., Kim S.K., Giacomini N., Boothroyd J.C., Sapolsky R.M. 2007: Behavioral changes induced by Toxoplasma infection of rodents are highly specific to aversion of cat odors. Proc Natl Acad Sci. 104: 6442-6447.

Wang H.L., Wang G.H., Li Q.Y., Shu C., Jiang M.S., Guo Y. 2006: Prevalence of Toxoplasma infection in first-episode schizophrenia and comparison between Toxoplasma-seropositive and Toxoplasma-seronegative schizophrenia. Acta Psychiatr. Scand. 114: 40-48

Webster J.P., McConkey G.A. 2010: Toxoplasma gondii-altered host behaviour: clues as to mechanism of action. Folia Parasitol. 57: $95-104$.

Yolken R.H., Bachmann S., Ruslanova I., Lillehoj E., Ford G., Torrey E.F., Schroeder J. 2001: Antibodies to Toxoplasma gondii in individuals with first-episode schizophrenia. Clin. Infect. Dis. 32: 842-844.

Yolken R.H., Dickerson F.B., Torrey E.F. 2009: Toxoplasma and schizophrenia. Parasite Immunol. 31: 706-715.

Received 30 May 2014

Cite this article as: Çelik T., Kartalci Ş., Aytaş Ö., Akarsu G.A., Gözükara H., Ünal S. 2015: Association between latent toxoplasmosis and clinical course of schizophrenia - continuous course of the disease is characteristic for Toxoplasma gondii-infected patients. Folia Parasitol. 62: 015. 